Itinéraires Itinéraires

Littérature, textes, cultures

\title{
Les cultures du chapitre : introduction
}

Cultures of Chapters: Introduction

Claire Colin, Camille Koskas et Jérémy Naïm

\section{CpenEdition}

Journals

Édition électronique

URL : http://journals.openedition.org/itineraires/8037

DOI : $10.4000 /$ itineraires. 8037

ISSN : 2427-920X

Éditeur

Pléiade

Référence électronique

Claire Colin, Camille Koskas et Jérémy Naïm, «Les cultures du chapitre : introduction », Itinéraires [En ligne], 2020-1 | 2020, mis en ligne le 13 novembre 2020, consulté le 13 novembre 2020. URL : http:// journals.openedition.org/itineraires/8037 ; DOI : https://doi.org/10.4000/itineraires.8037

Ce document a été généré automatiquement le 13 novembre 2020.

\section{cc) (†) $\odot$}

Itinéraires est mis à disposition selon les termes de la licence Creative Commons Attribution - Pas d'Utilisation Commerciale - Pas de Modification 4.0 International. 


\title{
Les cultures du chapitre : introduction
}

\author{
Cultures of Chapters: Introduction
}

Claire Colin, Camille Koskas et Jérémy Naïm

Ce dossier a pris sa source dans un colloque d'une semaine au Centre culturel international de Cerisy-la-Salle qui a eu lieu en juin 2018, et financé par l'ANR Chapitres : nous en sommes redevables à l'Association des amis de Pontigny-Cerisy, et en particulier à Édith Heurgon, que nous remercions ici pour son accueil, et pour nous avoir donné l'opportunité d'un échange d'idées dense et fructueux, dans un cadre exceptionnel. Pour soutenir l'Association et se tenir au courant de ses actualités, suivre le lien vers https://cerisy-colloques.fr/association/.

1 Jusqu'ici, la question du chapitre n'a pas été traitée en prenant en compte ses variations culturelles. À ce jour, l'ouvrage le plus marquant sur le sujet, et à bien des égards, l'ouvrage fondateur, celui d'Ugo Dionne (La Voie aux chapitres, Paris, Seuil, «Poétique », 2008), est d'abord un ouvrage de poétique. Dans la tradition genetienne, son auteur y invente une série de concepts permettant d'appréhender l'objet en tant qu'unité textuelle. Pourtant, dès l'introduction, Dionne effectue un pas de côté en présentant son ouvrage comme un travail de poétique "historique»: précision importante, qui renvoie au fait que le chapitre n'est pas seulement analysé comme unité linguistique, mais qu'il se rattache à des usages, à des pratiques, nécessairement divers et nécessairement historiques. On se souvient que dans Seuils ([1987] Paris, Seuil, «Point Essais », 2002), le livre de Genette qui contient les quelques pages que son auteur a consacrées au chapitre, l'introduction relève que l'ouvrage traite du « versant le plus socialisé de la pratique littéraire", ce qui explique qu'il «tourn[e] parfois inévitablement à quelque chose comme un essai sur les mœurs et les institutions de la République des Lettres»(p.19). C'est que le chapitre n'est pas, loin s'en faut, un élément textuel de même nature que, par exemple, la voix narrative. La génétique postéditoriale montre bien qu'il est, de toutes les unités du texte, l'une des moins stables : il apparaît, il disparaît au gré des éditions, des éditeurs, des choix de l'auteur et de format. Il est à la fois endogène et exogène à l'écriture, norme acquise comme le montre Ugo Dionne, au fil de l'histoire de l'écriture et de la circulation des textes. On 
comprend alors que Raphaël Baroni ait souligné, dans Les Rouages de l'intrigue, que le chapitre est une "science jeune " : la poétique qui se veut historique, dans le dialogue qu'elle implique entre les différentes histoires littéraires (histoire de l'enseignement, de l'édition, de la lecture, etc.), n'en est qu'au début de ses travaux.

Mais on sait que la démarche de Raphaël Baroni privilégie une approche presque anthropologique du texte. Celui-ci ne serait pas seulement une coïncidence de structures, objet immanent et figé, mais serait traversé par des fonctions "thymiques ", c'est-à-dire liées aux différentes émotions qu'il peut susciter. Son étude de La Tension narrative (Paris, Seuil, "Poétique», 2007) montre ainsi comment la narrativité s'organise autour des trois sentiments clefs que seraient le suspense, la curiosité et la surprise. Le chapitre, mise en silence du texte, clôture temporaire et temporelle, prendrait place ici dans sa capacité à rythmer les émotions du lecteur et à organiser son adhésion affective au texte. Rien d'étonnant dès lors à ce que, pour Philippe Hamon, le chapitre fasse partie « des principales macro-structures a priori de toute configuration", et qu'il relève de "procédés de mise en ordre quasi présémiotique du monde : le rythme, la mise en récit et la mise en liste " ("Ouverture ", Pratiques et poétiques du chapitre: 13). Dès lors qu'il y a chapitre, il y a actualisation de fonctions élémentaires de l'organisation discursive. Ce que ce numéro d'Itinéraires étudie, c'est la manière dont des genres, des médias et des arts différents actualisent le schème anthropologique commun de la division du récit en unités intermédiaires.

Il ne faudrait cependant pas, à trop généraliser, diluer l'identité du chapitre : qu'a de particulier cette division? Le mot est issu du latin classique capitulum, "petite tête ", qui désigne le chapeau ou le titre étendu permettant de se repérer dans le texte biblique. Dès le bas latin, le «chapitre » renvoie à une "partie d'un écrit » (TLFi), renforçant ainsi les fonctions de repérage ou de facilitation du parcours narratif que, plus tard, Du Cange (1678) signale dans son glossaire. Mais avec le temps, lorsque le chapitre perd son titre, qu'il disparait de la table des matières, qu'il se résume à un numéro parmi d'autres, que garde-t-il de ces fonctions qu'il avait dans le manuscrit dense du Moyen Âge? La "conquête » du roman par le chapitre, telle que l'a décrite Ugo Dionne, en a fait une norme de référence, qui appelle à l'adhésion ou au rejet. Rejet, par exemple, lorsque le nouveau roman, en quête d'un nouveau réalisme textuel, décide d'abandonner ce qui avait fait le roman balzacien au $\mathrm{XIX}^{\mathrm{e}}$ siècle (oubliant au passage que dans la dernière édition revue par Balzac, La Comédie humaine avait perdu la quasi-totalité de ses chapitres). Adhésion lorsque le chapitre sort du roman et vient diviser une bande dessinée (Watchmen), un film (Kill Bill), un jeu vidéo (Final Fantasy XV), voire une publicité : sur son site internet, Chanel propose ainsi de faire découvrir la maison "de l'intérieur " par le biais de vingt-huit chapitres, dont l'histoire serait à poursuivre. L'argument marketing relève du storytelling, il engage le consommateur dans la saga de la marque; mais il anoblit aussi l'image de marque en convainquant le client qu'il achète un objet aussi légitime qu'une œuvre littéraire. Car il est évident qu'il y a une légitimation par le chapitre, qui correspond, là aussi, à un positionnement socio-culturel. On voudrait ainsi faire justice au fait qu'il n'y a pas de division qui ne soit pas déjà discours : discours social, discours littéraire, "discours de la méthode " comme le suggère Georges Mathieu (2011: 221) ${ }^{1}$. Le chapitre est d'abord affaire culturelle, parce que la division renvoie à des positionnements sociaux, à des usages éditoriaux, à des mœurs littéraires et artistiques, en un mot à des pratiques inscrites dans des régimes historiques divers. Il nous faut supposer, dès lors, qu'il y a non pas 
une, mais des cultures du chapitre, des usages de la division qui renvoient à des sphères culturelles distinctes et potentiellement isolées : il est possible et même probable que la culture dramatique du XVII ${ }^{e}$ siècle n'ait rien de commun avec les normes de chapitrage du DVD à notre époque - pour reprendre deux exemples abordés dans ce numéro.

Le présent numéro invite ainsi à interroger dans leur diversité ces cultures du chapitre, en confrontant chaque fois la notion de chapitre à une notion parente mais qui ne le supplante pas nécessairement - tant le mot «chapitre » a essaimé d'un genre et d'un médium à l'autre : le chant dans l'épopée, l'entrée de journal, la nouvelle, le fragment, aussi bien que l'acte, l'épisode de série, le chapitrage d'un DVD, etc. En somme, l'ambition de ce dossier est d'explorer les variétés non romanesques (mais pas non narratives) du chapitre, et de comprendre comment ce mode de division a pu s'imposer aussi largement.

\section{Hors le roman, diviser la fiction}

Les trois premiers articles de ce numéro posent la question du chapitre dans les genres littéraires au-delà du roman. Ces genres fictionnels, qui n'ont pas, ou peu, été touchés par la «conquête du chapitre » telle que l'a retracée Ugo Dionne : respectivement, le théâtre, l'épopée, le recueil de nouvelles. Ces articles interrogent directement la théorisation de la coupure telle qu'on la comprend dans la littérature d'Ancien Régime. Marc Douguet met en balance le découpage théâtral avec la chapitration du roman. La comparaison est menée en termes de structure (analyse du contenu diégétique), mais aussi d'effet (quel type de suspense est produit par quel type de coupure). Marc Douguet rappelle l'opposition qu'a pu susciter l'idée même de coupure dans le théâtre classique, avant de mentionner que c'est l'utilisation du rideau, au XvIII ${ }^{e}$, mais surtout après le xIX ${ }^{e}$ siècle, qui a fini par rapprocher les divisions théâtrale et romanesque. Si la division du drame a été abondamment théorisée, Dimitri Garncarzyk souligne qu'à l'inverse, celle de l'épopée, pourtant objet théorique majeur aux $\mathrm{XVII}^{\mathrm{e}}$ et $\mathrm{xVIII}^{\mathrm{e}}$ siècles, est restée relativement pauvre. En tant que textes hérités, et donc édités, les épopées ont des divisions qui ressortent à la fois d'une structuration diégétique et d'une restructuration a posteriori. Ce que montre Dimitri Garncarzyk, c'est finalement que le chant est, à l'analyse, une unité très hétéroclite (elle peut comprendre plusieurs " unités rhapsodiques » selon le mot de son auteur), mais qu'il permet de retrouver une unité téléologique de l'action, et donc, paradoxalement, de structurer davantage le genre. Le recueil de nouvelles, lui, apparaît comme un genre immédiatement déstructuré. Si dans Les Mille et une nuits, la «nuit » peut apparaître comme une unité transversale à tout le recueil, dès la traduction de Galland, elle entre en tension avec l'unité "histoire ", souvent seule signalée dans la table des matières. Mais d'après l'étude de Jérémy Naïm, cette tension se résout historiquement en faveur de la nuit, et donc de l'unité du recueil : de plus en plus d'éditions des Nuits au Xix privilégient le marquage fort de la « nuit » dans le dispositif éditorial, quitte même à couper le récit là où il ne l'était pas dans les manuscrits d'origine. La «nuit » devient chapitre dès lors que le recueil est perçu comme une unité structurelle. 


\section{Aux limites du romanesque : le chapitre contesté ?}

6 La manière dont le chapitre se surimpose à des unités qui semblent avoir émergé plus directement du sujet de l'œuvre se retrouve dans les trois articles suivants: l'un interroge le chapitre comme manière romanesque de saisir les événements d'une vie, recueillis à l'origine en journal, chez Beauvoir; l'autre met en avant sa persistance surprenante, à l'inverse, dans des récits très courts à la forme hypersegmentée (Paulhan), voire dans les cut-up qui déconstruisent radicalement la forme romanesque. Hélène Baty-Delalande s'appuie sur l'ensemble de récits autobiographiques écrits par Simone de Beauvoir entre 1958 et 1981 (des Mémoires d'une jeune fille rangée à $L a$ Cérémonie des adieux) pour analyser les dispositifs capitulaires au sein des récits de soi, en soulignant l'importance que revêt le balisage du récit, dans un genre aimanté à la fois par la question de l'origine et de l'aboutissement: le chapitre présente-t-il les mêmes fonctions qu'au cœur des récits fictifs ? L'article souligne qu'il constitue bien un dispositif segmentant le récit, mais qu'il incarne aussi un lieu privilégié d'articulation, sinon de négociation, entre la totalisation partielle d'une expérience, et sa mise en perspective à l'échelle du récit tout entier. La notion de « chapitre » semble à l'inverse susceptible d'être questionnée dans les récits très courts écrits par Jean Paulhan entre 1916 et 1953. Découpés selon différents types de dispositifs, ceux-ci présentent tous une structure hypersegmentée, divisée en unités de très petites tailles. La construction du texte s'appuie sur une logique méthodique, qui voit sections et autres subdivisions s'enchaîner selon un ordre rigoureux, objet de remaniements minutieux, comme le montrent les plans et brouillons conservés dans le fonds Jean Paulhan à l'IMEC. L'article de Camille Koskas interroge donc les liens que le chapitre entretient au sein de ces récits avec d'autres formes de divisions narratives et argumentatives: segments, sections, fragments. Il questionne également le sens de ces petites unités à l'échelle de l'œuvre entière, de nature essayistique ou fictionnelle, en cherchant à montrer que cette inclination pour l'extrême fragmentation des textes s'inscrit dans une dialectique avec la quête d'unité qui anime l'ensemble de l'œuvre.

7 Pareille dialectique est exhibée plus explicitement encore dans l'article de Clémentine Hougue, consacré au chapitre dans plusieurs œuvres de John Dos Passos, William Burroughs et J.G. Ballard. Elle montre que les pratiques de montage et de collage interfèrent nécessairement avec les fonctions premières de division narrative d'un chapitre désormais fragmenté et pulvérisé. Cette déconstruction sert l'entreprise politique au cœur de ces romans : il s'agit d'émanciper le lecteur de ses habitudes, nées en particulier des discours médiatiques convenus et asservissants, et de le pousser à s'approprier l'œuvre par une lecture personnelle et assumée. Déstabilisé, remis en question, le chapitre reste cependant dans ces textes la pierre angulaire d'une réflexion sociétale : il fournit une structure qui ne disparait pas, mais est mise en concurrence avec d'autres, ce qui lui accorde une certaine réflexivité.

\section{Le devenir du chapitre : d'un médium à l'autre}

8 La question de la persistance ou au contraire de la contestation, voire du dépassement du chapitre se pose de manière particulièrement visible dans le cas de l'adaptation, qui permet de comparer plusieurs états d'une œuvre sur des supports différents. Dans l'analyse qu'il consacre à Georges Darien et en particulier au roman Le Voleur (1897), 
Aurélien Lorig montre dans un premier temps comment le chapitre constitue une porte d'entrée privilégiée pour saisir la poétique profonde de l'œuvre, où se joue l'influence de plusieurs genres, en premier lieu le roman-feuilleton, sans qu'un seul domine véritablement. Lorsqu'il adapte en 1967 le livre, Louis Malle semble justement avoir saisi cette importance des chapitres et de ce qu'ils peuvent traduire pour restituer l'essence profonde $\mathrm{du}$ projet initial tout en s'inscrivant dans le genre cinématographique. En effet le cinéaste comprend la force d'effraction contenue dans le roman à travers des choix précis d'enchaînements de séquences, où le séquençage sans transition permet de rappeler le refus des conventions et des barrières au cœur des convictions de Darien. De la même façon, l'adaptation en bande dessinée conçue par Bernard Seyer (1986), si elle ne peut œuvrer dans la logique du chapitre court et des enchaînements romanesques propres à la veine du roman-feuilleton qui innerve le roman de Darien, parvient toutefois à en traduire l'esprit par le resserrement autour des scènes les plus propres à susciter l'attente et l'émotion du lecteur et par l'utilisation d'une certaine dramatisation dans le dessin. Les deux adaptations ont donc tenté de respecter le projet de Darien, tout en cherchant à appliquer aux modalités du genre concerné, cinématographique pour l'un, bédéique pour l'autre, la plasticité qui est la caractéristique première du Voleur, et d'abord à travers la poétique des chapitres dans l'œuvre.

9 L'article d'Aude Leblond revient sur la distinction entre chapitre et épisode et pose la question du devenir des chapitres dans deux adaptations audiovisuelles de deux cycles littéraires, Harry Potter pour le support cinématographique et Game of Thrones pour la série télévisée. Malgré une volonté d'afficher une grande fidélité dans l'adaptation, il apparaît que les chapitres de ces œuvres ne sont pas directement transposables, mais font l'objet d'une réécriture conditionnée par le support prévu, et donnent lieu à une sélection, un allongement ou une redistribution des événements, ainsi qu'une concentration des éléments saillants. Aude Leblond démontre également que la question de l'adaptation du chapitre sous forme audiovisuelle permet de s'interroger de façon renouvelée sur sa poétique, et en premier lieu sur ses constituants. Elle souligne en effet que si les termes de paragraphe et de séquence peuvent être retenus pour l'étude du texte, celui de scène (qui requiert la présence d'au moins deux unités parmi celles de temps, de lieu ou de personnel) est davantage exploitable pour analyser la transposition à l'écran. En effet, chapitres comme épisodes sont construits autour de scènes, moins nombreuses cependant pour les chapitres. L'étude de la clôture en revanche ne montre pas de distinction nette entre chapitre et épisode, car elle occupe indistinctement selon les deux supports la fonction d'offrir un temps de bilan tout comme de relancer l'intrigue. Ainsi, l'adaptation des chapitres est aussi source de nouvelles potentialités narratives et promet une relance du plaisir de la fiction.

Les cas d'adaptation envisagés ici montrent somme toute une volonté de fidélité au support initial, et partant à son mode de structuration.

\section{Extension du domaine du chapitre : cultures visuelles et audio-visuelles}

11 Quand on s'affranchit plus nettement du régime textuel, le modèle du chapitre peut être repris comme référence, à la façon d'un modèle normatif soulignant l'importance des fonctions narratives et poétiques, voire anthropologiques qui lui sont associées, 
comme on l'a vu dans les exemples publicitaires précédemment évoqués. Ainsi, Alain Boillat souligne dans l'étude qu'il consacre au chapitrage dans les DVD l'importance et la diversité de cette forme de balisage, moyen de se réapproprier une œuvre cinématographique, tant pour l'éditeur qui choisit les différents chapitres que pour le spectateur, qui peut entrer dans l'œuvre à partir d'une scène de son choix, et reconquiert par là une maîtrise du récit. De la même façon, Raphaël Luis souligne l'importance du chapitre dans le comic book The Sandman, écrit par Neil Gaiman : il montre d'une part son désir d'anoblir son roman graphique en l'exhibant comme une œuvre littéraire, et d'autre part, à travers la multiplicité des formes chapitrales, il laisse le lecteur libre de choisir ses modalités de lecture. Le chapitre devient ainsi dans le domaine de la bande dessinée à la fois un lieu de diversification générique et un espace de liberté.

Mais cette présence du chapitre au-delà de la littérature, montrant combien il imprègne nos usages de tout ce qui s'apparente à un récit, peut être également questionnée. Devenu usuel, ce terme de "chapitre » est peut-être trop rapidement utilisé quand finalement d'autres s'avèrent plus pertinents. Ainsi les articles d'Anaïs Goudmand et de François Jost, tous deux consacrés aux séries télévisées, s'attachent à montrer combien l'utilisation du terme « chapitre » est à prendre avec prudence : Anaïs Goudmand étudie le cas des séries proposées par la chaîne Netflix, et montre que le chapitrage, pourtant affiché en tant que tel, n'est pas une norme, mais qu'il implique une grande variété d'intentionnalités et de modes de réception. De la même façon, François Jost s'interroge sur la possibilité de parler de chapitres pour les séries télévisées et insiste sur la nuance qu'il convient d'apporter à la réponse, puisque les cas de chapitrage sont bien présents, mais coexistent avec une construction qui s'appuie davantage sur la dramaturgie théâtrale. Cette nécessaire nuance souligne dans tous les cas la place qu'a pu prendre le chapitre, dans son appellation et dans sa fonction attendue, pour des supports autres que littéraires. Néanmoins le chapitre, par sa seule présence, pose la question de sa fonction et de sa force, réelle ou supposée.

Peut-être cela pourrait-il expliquer la présence de chapitres dans le cas tout à fait exceptionnel des narrations interactives. L'analyse qu'Olivier Caïra consacre au champ d'étude innovant que constitue le chapitre dans la fiction interactive (jeux de rôle comme jeux vidéo) le dote de nouvelles possibilités. Le chapitrage de ces jeux traduit d'une part l'héritage du texte narratif, l'instauration d'un rythme et la division du récit, et d'autre part tout le potentiel d'innovation que peut offrir ce type de nouveau récit, qui entraîne une véritable métamorphose de la division narrative quand on étudie ses fonctions spécifiques dans l'organisation d'un jeu. Si au premier abord le chapitrage des jeux obéit à des contraintes similaires à celles du roman (gestion dans le récit du rythme et des composantes de l'intrigue en premier lieu), l'interactivité propre à ce support conduit nécessairement à modifier la clôture chapitrale, l'interruption en plein combat étant ainsi difficilement envisageable durant une partie de jeu vidéo ou de jeu de rôle, à la différence du texte narratif. En outre, la fiction ludique peut entraîner une modification de la coupe chapitrale pour des raisons ludiques : le goût du jeu ou de la performance l'emporte alors sur la logique de l'intrigue, conduisant à répéter un même segment narratif et à faire du chapitre une unité à part entière. Mais au-delà de ces questions de coupe, les chapitres dans la fiction interactive ne sont plus du ressort de l'auteur mais bien des joueurs, qui décident de leur disposition dans l'intrigue ainsi que 
de leur nombre, ces décisions entraînant nécessairement une navigation d'un chapitre à l'autre, le fil narratif unique et fixe cédant la place à la porosité et à la multiplicité.

Alors même qu'il est longtemps resté un impensé de la théorie littéraire et qu'il demeure le plus souvent invisible à nos yeux, le chapitre apparaît donc comme un médium capable non seulement de susciter une réflexion poétique, mais également culturelle et anthropologique. Diviser, ordonner sont autant d'actes fondateurs pour toute société, pour ses pratiques culturelles: étudier le chapitre permet ainsi de questionner nos usages du récit, ceux du passé comme ceux à venir, tout comme nos rapports à la fiction dans tous ses états. Sa fonction initiale de simple repère dans un texte est de cette façon nettement élargie, faisant de lui un possible témoin marquant d'une époque.

\section{NOTES}

1. Georges Mathieu, «Étudier la division d'un roman en chapitres : quelques réflexions méthodologiques ", dans Sylvie Triaire et Patricia Victorin (dir.), Deviser, diviser: pratiques du découpage et poétiques du chapitre de l'Antiquité à nos jours, Montpellier, PULM, 2011.

\section{AUTEURS}

\section{CLAIRE COLIN}

Claire Colin enseigne dans le secondaire. Elle a soutenu en 2013 une thèse de littérature comparée consacrée à l'événement dans la nouvelle contemporaine et a co-dirigé le volume Pratiques et poétiques du chapitre du xixe au xxie siècle (PUR, 2017).

\section{CAMILLE KOSKAS}

Sorbonne Université

JÉRÉMY NAÏM

Lycée Watteau, Valenciennes 